\title{
Nuclear data for fusion applications
}

\author{
R.A. Forrest ${ }^{\mathrm{a}}$ \\ Euratom/UKAEA Fusion Association, Culham Science Centre, Abingdon, Oxon, OX14 3DB, UK
}

\begin{abstract}
Achieving the economically viable release of energy by the fusion process has proved a major scientific and technological challenge. The nuclear reactions involved in the fusion process are well understood, but the interaction of the neutrons with the materials surrounding the plasma is an area of current research. The neutrons have energies larger than those experienced in fission and this has been a driver to extend the types and amounts of nuclear data. Designs for fusion devices require neutron and photon transport calculations to generate the spectra required to provide the nuclear responses. Such calculations require cross section data for all elements present in the device. ITER is the next step on the road to demonstrating the commercial generation of electricity. The importance of nuclear data for ITER was realized many years ago, which led to the release of FENDL-1 in 1995. This contained both general and special purpose files; an example of the latter is activation. Development of these files focused on completeness - including $\gamma$ production and covariances; the files generally had an upper energy limit of $20 \mathrm{MeV}$. The types of data in the fusion relevant libraries are sufficient for all foreseeable fusion devices. However, the transition in fusion research from plasma physics towards technology requires an understanding of the materials damage caused by the intense fluxes of neutrons that will be found in power plants. To gain this knowledge a materials test facility, IFMIF, is required. This is planned to operate in parallel with ITER and will use accelerated beams of deuterons striking a flowing lithium target to generating an intense neutron field. The neutrons will mostly be fusion relevant, but there will be a high-energy tail extending up to $\sim 55 \mathrm{MeV}$. These high energy neutrons further extend the amount of nuclear data required and have stimulated much theoretical and experimental work covering the energy range $>20 \mathrm{MeV}$. Examples of recent general purpose evaluations, new activation libraries and benchmarking are described and the challenges for nuclear data for fusion discussed.
\end{abstract}

\section{Introduction}

Fusion is the physical process that powers the sun and stars and it is a dream of mankind that it should be harnessed for environmentally attractive and abundant energy production on earth. The process in the sun is the proton-proton cycle where two protons react to form deuterium, which reacts with a proton to form ${ }^{3} \mathrm{He}$. Two ${ }^{3} \mathrm{He}$ then react to produce ${ }^{4} \mathrm{He}$. The reaction rate of the first step is extremely slow, but the huge number of protons in the core ensures that this is a feasible energy production method. For terrestrial energy production this cycle cannot be used, but by using reactions between heavier hydrogen isotopes, deuterium (D) and tritium (T), the scheme of D-T fusion is feasible.

The sun uses gravitational confinement to give the protons sufficient time to react; again a different method, magnetic or inertial confinement is required on earth. These facts have been known for a long time and there was unwarranted optimism in the late 1950's that fusion power could be quickly achieved (see the press announcements about Zeta [1]). However, a great deal of effort on plasma physics has been required to reach the point where now we have confidence that a device can be built to release large amounts of fusion power $(\sim 500 \mathrm{MW})$ about $10 \times$ more than input. ITER [2] is this device and it is appropriate that this plenary talk on fusion, the first at a Nuclear Data conference for several years should be taking place a short distance from where ITER will be built.

${ }^{a}$ Presenting author, e-mail: robin. forrest@ukaea.org.uk
It is my task in this paper to give some of the history of how research in fusion has driven the development of nuclear data, how it is still an important application today and how in my personal opinion nuclear data needs to adapt to meet the challenges of fusion as an energy source in the medium term future.

It is important to stress the differences between the nuclear data needs of fusion and fission and how these have driven the development of fusion specific data files such as FENDL. ITER and other fusion devices are only one part of the roadmap for the commercial production of electricity by fusion. Although the plasma physics required for a fusion power station has reached the point where its predictions can be tested on ITER, the technology aspects are less well developed. An important area is the response of materials to the intense neutron fluences, much greater that in ITER, that will be experienced in a power plant. In order to test and qualify materials such as the steels that will be used for the structure it is necessary to build a materials test facility. IFMIF [3] is planned to operate in parallel with ITER and this device has its own set of nuclear data needs. Activation data are of primary importance for fusion technology, whereas for fission they are only secondary. Activation data have thus been another driver for nuclear data development.

Data files rely on model codes and the use of these for the evaluations of fusion specific data are described. The validation of both general purpose and activation libraries is vital and work in this area is discussed. The continuing increases in computer power and the rise of the web for 
viewing data suggest a new direction for nuclear data. This involves the efficient handling of large amounts of data leading towards more complete libraries that can be used as input for sophisticated neutronics design tools. It is argued that to reach this point significant investment in nuclear data is both very important and timely in view of the continuing energy and environment debate.

\section{Fusion}

Fusion has many advantages as a power source: essentially unlimited fuel, no generation of greenhouse gases, no major accidents and no production of radioactive "ash". However, radioactivity is involved as neutrons will activate components of the plant. Indications [4] are that it will be a competitive means of electricity generation. A roadmap [5] for fusion research showing the connections between the current experiments, ITER, IFMIF, DEMO and commercial power plants has been produced. This shows ITER and IFMIF operating in parallel and providing information needed for the design of DEMO which should be ready for start of construction in about 30 years from now.

ITER (The way) has the objective of demonstrating the scientific and technological feasibility of fusion. It is an international collaboration of countries representing over half the world's population. ITER has the dimensions of a power plant but will not generate electricity. At the present time the main work is in setting up the project organisation and resolving some remaining technical issues raised by the design review. Construction is expected to start at the beginning of 2009.

IFMIF has the objective of qualification of candidate materials up to the full lifetime of anticipated use in fusion power plants and the validation of the existing data generated from fission reactors and particle accelerators. It will use two deuteron beams $(125 \mathrm{~mA})$ accelerated to $40 \mathrm{MeV}$ and striking a flowing lithium target to generate an intense neutron field. The main reactions are the stripping reactions ${ }^{7} \mathrm{Li}(\mathrm{d}, 2 \mathrm{n}){ }^{7} \mathrm{Be}$ and ${ }^{6} \mathrm{Li}(\mathrm{d}, \mathrm{n})^{7} \mathrm{Be}$, although absorption reactions mean that the neutron spectrum has a high energy tail extending to $55 \mathrm{MeV}$. The test cell behind the target has regions of high flux $(<20$ $\mathrm{dpa} / \mathrm{y}$ in 0.51$)$, medium flux (1-20 dpa/y in 61) and low flux $(>1 \mathrm{dpa} / \mathrm{y}$ in 81 ) where samples can be irradiated. As part of the Europe-Japan agreement the EVEDA phase of IFMIF is under way and will lead to the construction of part of the accelerator.

\section{Fission and fusion nuclear data needs}

In a fission power plant the most important nuclear event is the fission of the actinide fuel producing fission products and energy. The neutrons are used "locally" within the fuel to initiate another fission event either directly or following moderation. Capture of the neutrons removes them and stops them playing a useful role in the chain reaction. Neutrons can escape into the outside world, but this is minimised by shielding. This extremely simplified view emphasises that the dominant reactions are $(\mathrm{n}, \mathrm{f})$ and $(\mathrm{n}, \gamma)$ on actinides and that because the important nuclear reactions are localised in the fuel and moderator the distributions of emitted particles are not very important.

In contrast for fusion there are two main regions of nuclear events. The first in the plasma consists primarily of reactions of hydrogen isotopes producing energetic $(14 \mathrm{MeV})$ neutrons. These neutrons then have to travel a significant distance through the first wall to reach the blanket. Here they react with "multipliers" like beryllium or lead through the $(\mathrm{n}, 2 \mathrm{n})$ reactions and then with the lithium "breeder" to produce tritium through the $(\mathrm{n}, \alpha)$ and $\left(\mathrm{n}, \mathrm{n}^{\prime} \alpha\right)$ reactions. The extended geometry between these two regions means that how the neutrons travel and in which ways they scatter and react are important and thus knowledge of the angular distributions is required. The reactions also determine how the materials of the fusion device are activated. Shielding is important, not only to stop neutrons reaching the outside world, but also to protect the superconducting magnets which to operate must receive an extremely low neutron dose. It can be seen that elastic, inelastic scattering, $(n, 2 n)$, tritium production, angular distributions, gamma production (for heating) and a range of activation reactions are now the important nuclear data issues.

Thus while fission data tends to focus on a range of actinides, especially $\mathrm{U}$ and $\mathrm{Pu}$ and fission products, fusion must address the light elements $\mathrm{H}, \mathrm{He}, \mathrm{Li}$ and $\mathrm{Be}$ (plasma reactions, tritium production and neutron multiplication); structural elements Ti-Ni; C, Mo and W for first wall armour; $\mathrm{Cu}, \mathrm{Nb}$ and $\mathrm{Sn}$ for magnets and $\mathrm{Pb}$ as a multiplier. In the future $\mathrm{SiC}$ may replace steel. Thus the range of elements important for fusion is wider than for fission and of course the neutron energy around $14 \mathrm{MeV}$ is significantly larger that the $2.5 \mathrm{MeV}$ neutrons produced in fission.

When the IFMIF device is considered there are needs for a detailed description of the neutron production from $40 \mathrm{MeV}$ deuterons on lithium and for neutron interaction data up to $55 \mathrm{MeV}$ on the structure and materials undergoing testing. In addition because of the high deuteron current, losses in the accelerator can cause deuteron-induced activation. There will be a wide range of elements in the lithium loop due to corrosion and these can also interact with the deuterons producing activation. In the initial phase of IFMIF testing beams other than $\mathrm{D}^{+}$, either $\mathrm{H}_{2}^{+}$or $\mathrm{H}^{+}$may be used and there is therefore a need for proton-induced activation data.

It is relevant to note that in the case of a fission power plant the scope for changing the "impact" is rather limited as it is determined by the physical process of fission. By impact is meant the effects of an accident, the doses to workers during normal operation and wastes arising from decommissioning. In a fusion power plant the choice of materials is crucial in determining all aspects of the impact and thus explains the much greater emphasis on activation data in fusion work.

There are of course many topics which are common to both fission and fusion studies. These include the importance of neutronics calculations, the need for comprehensive covariance data to enable detailed sensitivity studies to be carried out, decay data for all relevant nuclides and a range of dosimetry data to enable neutron spectrum unfolding. These two energy production technologies also have a range of links with medical nuclear data, accelerator applications and basic science needs, especially astrophysics. 
Table 1. Information from previous Nuclear Data conferences (with date). The percentage of papers in the fusion session and its title are shown.

\begin{tabular}{|c|c|c|}
\hline Conference & Fusion papers $(\%)$ & Fusion session \\
\hline Antwerp (1982) & 8.7 & $\begin{array}{l}\text { Nuclear data pertaining } \\
\text { to fusion reactors and } \\
\text { intense neutron sources }\end{array}$ \\
\hline Santa Fe (1985) & 13.5 & $\begin{array}{l}\text { Differential and integral } \\
\text { data for fusion reactors }\end{array}$ \\
\hline Mito (1988) & 13.4 & $\begin{array}{l}\text { Differential and integral } \\
\text { data for fusion reactors }\end{array}$ \\
\hline Jülich (1991) & 12.6 & $\begin{array}{l}\text { Nuclear data for fusion } \\
\text { reactor technology }\end{array}$ \\
\hline Gatlinburg (1994) & 9.5 & $\begin{array}{c}\text { Nuclear data for fusion } \\
\text { applications }\end{array}$ \\
\hline Trieste (1997) & $13^{*}$ & No dedicated session \\
\hline Tsukuba (2001) & 5 & $\begin{array}{c}\text { Applications to fusion } \\
\text { technology }\end{array}$ \\
\hline Santa Fe (2004) & 4.3 & Fusion energy \\
\hline
\end{tabular}

* Papers on fusion estimated.

\section{Fusion nuclear data}

Prior to considering how nuclear data for fusion has developed it is interesting to look back at some of the previous conferences in this series at the treatment of fusion data. Table 1 shows some details. At first sight it appears that fusion data has become less important since a peak in the mid 1980's; however, this may be because fusion data has increasingly been treated as mainstream data rather than as a separate topic. Also the emphasis on the sessions has moved from "Reactors" to "Technology" to "Energy", i.e., from speculation to mature energy production.

The importance of materials research has already been mentioned in relation to IFMIF; note how the Antwerp fusion session mentions "intense neutron sources" and the paper at Jülich by the late S. Cierjacks [6] should be recalled stressing the need to understanding both materials damage and "low activation materials". Today the theoretical study of material damage is a very important field in its own right.

\subsection{FENDL}

Looking back to the early 1980's the focus for fusion nuclear data was on: the light particle reactions in the plasma; tritium production; the measurement of gamma production; the $(n, 2 n)$ reaction on $\mathrm{Be}$ and $\mathrm{Pb}$ and integral benchmarks for fusion relevant materials. It was increasing recognised that the existing data files such as ENDF and JEF were inadequate for fusion studies, needing more DDX data and data at energies above $10 \mathrm{MeV}$. There were also many measurements of neutron activation, especially for gas production reactions such as $(n, t)$ and on finding systematic formulae to describe a range of reaction types.

In response to the fusion specific needs, and with the start of ITER discussions, the IAEA took the lead with a series of meetings focussing on the data needs of fusion. These defined the requirements of a fusion nuclear data library and ultimately led to FENDL-1 [7] in 1995. Although the product was extremely important, even more so was the process whereby it was created. This involved extensive comparison of evaluated data files from regional libraries (ENDF, JEF, BROND, JENDL, CENDL) and the selection of the best and most fusion relevant data from each. Such comparison is today commonplace, at the time it was groundbreaking. In addition to the general purpose library for neutronics, special purpose files on charged particle data, activation, dosimetry and decay data were included in FENDL-1. The IAEA NDS took responsibility for the library construction and processing producing multi-group and Monte Carlo libraries. It should be noted that the evaluations on which FENDL is based were not funded by the IAEA but were volunteered by the regionally funded projects. Benchmarking of FENDL-1 was extremely important in identifying shortcomings and the FENDL-2 library [8] was produced in 1997-1998. This also contained new versions of the special purpose files. The benchmark collection covers the materials $\mathrm{Li}, \mathrm{LiF}, \mathrm{Be}, \mathrm{Be}-\mathrm{Li}, \mathrm{C}, \mathrm{CF}_{2}$, N, O, Teflon, Al, Si, Ti, Cr, Mn, Fe, Stainless steel, steelwater, $\mathrm{Co}, \mathrm{Ni}, \mathrm{Cu}, \mathrm{Zr}, \mathrm{Nb}, \mathrm{Mo}, \mathrm{W}, \mathrm{Pb}, \mathrm{Pb}-\mathrm{Li}, \mathrm{Pb}-\mathrm{Li}-\mathrm{C}$. In 2004 the current version FENDL-2.1 was released [9] (http://wwwnds.iaea.or.at/fendl21/index.html). The number of materials in the general purpose library is extended to 71 , while for the dosimetry file it is recommended to use IRDF-2002 [10] and for the activation file EAF-2003 [11] = JEFF3.0/A [12].

Apart from this fusion specific library, nuclear data relevant to fusion can now mostly be found in the standard libraries. The European Fusion File which was last distributed as a separate file as EFF-2.4 [13] has become part of JEFF [14]. ENDF [15] and BROND [16] have always included fusion relevant data; and while the JENDL-FF [17] still exists, the last release is old (1999) and JENDL-3.3 [18] contains fusion data. The exception to the rule that fusion data are to be found in the standard data files is for activation. The next section explains why activation data are different.

\subsection{Activation data}

Activation data only contain cross sections as a function of energy, $\sigma(E)$. However, because the impurities in a material rather than the base elements tend to dominate activation properties, it is necessary to include all elements in the libraries. Experience has shown that multi-step pathways to radionuclides are important and for many reactions on unstable nuclides contribute. This means that cross sections for a range of radioactive targets are necessary. For fusion devices an upper energy of $20 \mathrm{MeV}$ is sufficient, but for IFMIF studies this limit needs to extend to about $60 \mathrm{MeV}$.

Activation calculations determine how much of a radionuclide is formed following irradiation. Consequently the elastic cross section is not required and inelastic data are only needed when the daughter has an isomeric state. There is therefore considerable freedom in the choice of data source for reactions on a particular target. It is common that these will come from a range of sources and in some cases this is also true for different energy regions of a single reaction. To make the above points more concrete some statistics for recent European Activation 


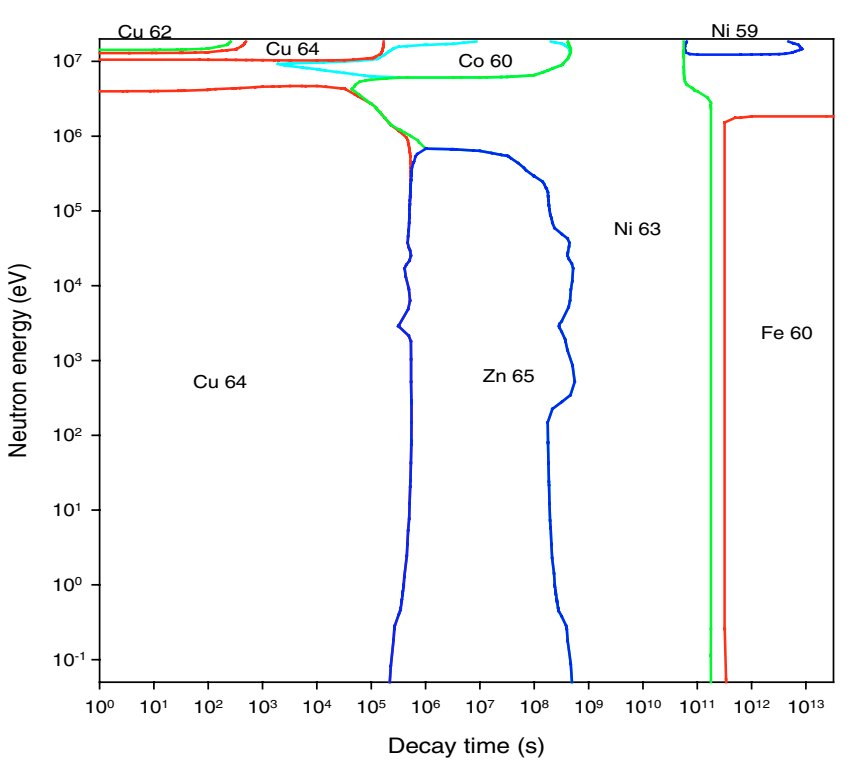

Fig. 1. Activity importance diagram for $\mathrm{Cu}$. In a region labelled by a nuclide the nuclide contributes at least $50 \%$ of the activity.

File libraries are given. EAF-2003 [11] extended to $20 \mathrm{MeV}$ and contained cross section data for 12,617 reactions on 774 targets. EAF-2007 [19] extends to $60 \mathrm{MeV}$ and contains cross section data for 65,565 reactions on 816 targets. For both of these EAF libraries most reactions have not been measured and the libraries therefore rely to a large extent on model calculations.

An extremely important question for such libraries is to find which of these reactions are really important in determining the activation properties. Lists of important reactions have been available for many years [20], but these depended on the neutron spectrum and on which quantities (activity, $\gamma$-dose rate, heating) were considered important in the study. Consequently the lists changed with time and were only a partial guide to which reactions should be studied in more detail.

Recently the use of importance diagrams [21] has improved the situation. For a particular quantity, e.g., activity, the dominant nuclides at various cooling times are determined following irradiation by neutrons of a particular energy. Repeating such calculations at other energies allows a diagram to be constructed with regions showing where particular nuclides contribute at least 50\%. Figure 1 shows such an importance diagram for activity in copper. Thus for neutron energies $<0.5 \mathrm{MeV}$ and for decay times $<5 \times 10^{8} \mathrm{~s}$ and $>2 \times 10^{5} \mathrm{~s}{ }^{65} \mathrm{Zn}$ dominates the activity. The regions are almost independent of neutron flux and thus the diagram gives a universal picture of the nuclides important for the quantity. The pathways responsible for the production of the various dominant nuclides can be found at particular energies.

An extensive study of the activation of all elements has been reported [22,23], and from this the important reactions can be identified. For the EAF-2003 library only 1,314 of the 12,617 reactions are necessary to produce the 754 radionuclides that dominate the various properties. Of these important reactions some are well measured, and a definitive list of

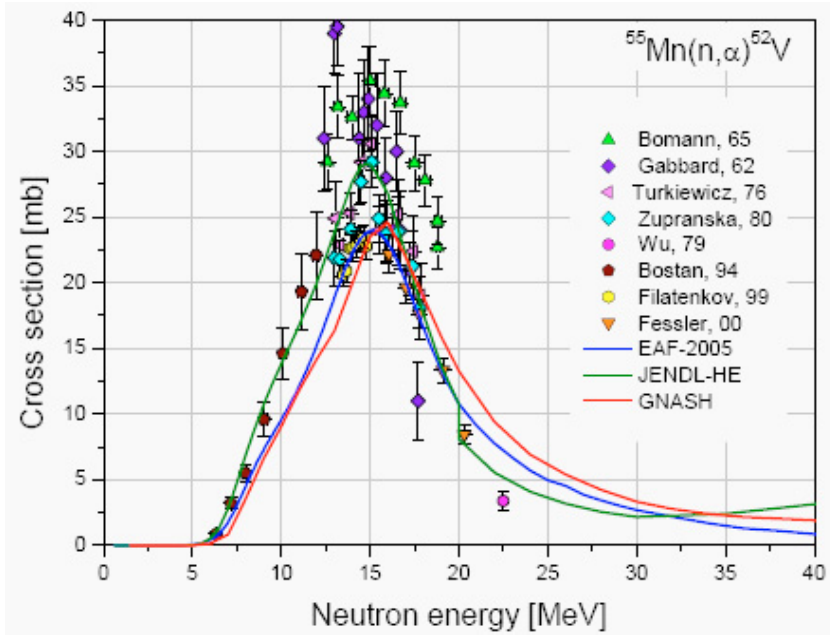

Fig. 2. Evaluation of the ${ }^{55} \mathrm{Mn}(\mathrm{n}, \alpha)^{52} \mathrm{~V}$ reaction (GNASH) compared with EXFOR data.

reactions requiring additional study, either by measurement or calculation has been found [24].

\subsection{Evaluations}

Evaluation requires a model code and experimental data. The new generation of model codes such as TALYS [25] and EMPIRE [26] are extremely comprehensive and are capable of generating all the required quantities as an ENDF formatted file. The use of recommended sets of parameters such as RIPL2 [27] enables calculations to be done reliably. Experimental data from EXFOR can be compared with the model calculations and used to improve them. For fusion it is important that the evaluation extend to $60 \mathrm{MeV}$, although most extend to 150 or $200 \mathrm{MeV}$ as the same physical models can be used and the extra work is minimal. Examples of recent evaluations are for the isotopes of $\mathrm{W}$ [28], ${ }^{181} \mathrm{Ta}$ [29] and ${ }^{55} \mathrm{Mn}$ [30]. Figure 2 shows part of the cross section data for one reaction on ${ }^{55} \mathrm{Mn}$, that actually extends to $150 \mathrm{MeV}$.

An item of increasing importance is covariance data. Only a small number of the existing evaluated files contain reasonably complete covariance data. Progress is being made on theoretical calculations based on estimates of the uncertainty in the model parameters [31]. A more traditional approach based on the spread of experimental points and use of a Bayesian code [32] is able to generate the covariance data and greatly reduce the variance data for well measured reactions. Such data can then be added to an evaluated file.

\subsection{Benchmarking}

Benchmarking of evaluated files allows the data to be validated for routine use, but also indicates where it can be improved. The extensive benchmarking of the FENDL files was mentioned above, and all fusion relevant files considered for inclusion in JEFF undergo similar testing [33]. An important extension of this is to consider not just a simple geometry (shell or slab) and material (iron) but a "mock-up" of a device such as a test blanket module (TBM). TMB's will be inserted in the ITER ports and used to test the various methods of 


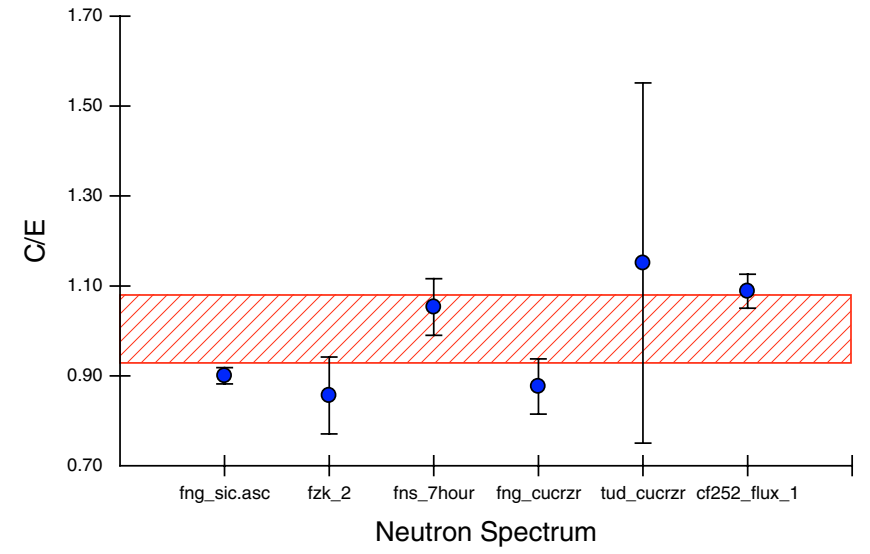

Fig. 3. $\mathrm{C} / \mathrm{E}$ values for ${ }^{65} \mathrm{Cu}(\mathrm{n}, 2 \mathrm{n}){ }^{64} \mathrm{Cu}$, the band represents the uncertainty in the EAF-2007 library.

generating tritium in the blankets of future power plants. The work at ENEA [34] with a mock-up relevant for a HCPB module has brought together many measurement and calculational techniques and has realised a critical result. Considering tritium production it was found that the calculated result was less than the measured result $(\sim 0.95)$ giving confidence that the estimates of tritium economy for fusion power plants are conservative.

\subsection{Validation of activation data}

The methodology used for validation of activation libraries such as EAF is less well known than the benchmarking of transport files and is discussed below in some detail. Measurements of activity following irradiation in a known neutron spectrum are made (E) and compared with calculations using the data library $(\mathrm{C})$. Although it is possible to report $\mathrm{C} / \mathrm{E}$ values for the activity of various nuclides it is preferable to associate the measurement with the average value of the cross section for production of the nuclide in the spectrum. This has been done for the study of EAF-2005 [35], and an example of a validated reaction is shown in figure 3 . The measurement error bars overlap the library error band signifying good agreement. Note that there must also be satisfactory agreement with the differential data before a decision can be reached on the reaction status. Further discussion is given in ref. [36], which also discusses a statistical method (SACS) that can be used to test activation libraries and which is particularly important for reactions with no measurements.

\subsection{Data needs}

The focus of most current fusion nuclear data work is on the near term - ITER and IFMIF. The discussion above has indicated the large number of materials that are present in these devices. There are several papers $[37,38]$ that present in more detail than is possible here the needs for these devices. The main requirement is for complete evaluated files extending at least up to $60 \mathrm{MeV}$, completeness meaning that angle-energy coupled emission data, gamma production and covariance data are present. To enable good evaluations the experimental database needs to be extended, particularly by a number of high-precision cross section measurements. These should be focused on those reactions with known discrepancies or where new data are needed to guide accurate model calculations. It is of particular importance that data at energies above $20 \mathrm{MeV}$ be available as many models and evaluation agree around $14 \mathrm{MeV}$ but then diverge at higher energies.

New measurements and evaluations are planned in the next few years under a "business as usual" scenario. While these are important in addressing known deficiencies I believe that it is also necessary to also pursue a more radical approach. This is suggested by several developments: cheap computer power, both in speed and storage; interactive data viewing capability such as JANIS [39]; discussions on replacing the regional files with a "universal file"; the desire for convergence of the general and special purpose libraries and discussions on new data formats. A new approach has been pursed for the activation libraries and this is described below as it can act as a "model" for the general purpose files.

Data belongs in a database, where standard methods such as SQL allow it to be sorted, viewed and manipulated. However, the ENDF formatted files are still at the heart of nuclear data work because of the large investment in processing and application codes. It is for this reason that radical changes in format have been resisted and this is likely to continue in the medium term. But if formatted text files remain there is no reason that the data must remain in that form during the development phase. The EAF files are text files (slightly nonENDF standard) as required by a FORTRAN application like FISPACT [40]. They are produced from many data sources ( 50) using the SAFEPAQ-II application [41]. This uses a modern programming language (Visual Basic) giving an intuitive interactive user interface and stores all the data within relational databases (Access). Once in the database all data can be compared and manipulated (for EAF-2007 55,000 modifications were made) and then when complete the final data are written as text files. SAFEPAQ-II keeps the data consistent when changes are made, e.g., removing a cross section point also changes the interpolation data table.

For the general purpose files a similar but more sophisticated evaluation tool is needed - interactive, using relational databases and as changes are made by the user working in the background to keep the data consistent. A simple example of its use would be to replace one reaction channel with data from another source and to ensure that the totals and the emission data remained consistent. Prior to using this tool it is essential that complete files are generated using a modern code such as TALYS for a wide range of targets. These should include all the stables, but ultimately could also cover the same list of targets as the activation libraries. Data in this "base" file could then be replaced by better data from the existing evaluated libraries producing a first phase improved library. This should then be benchmarked and iterative improvements made. Setting up such an infra-structure of an evaluation tool and producing the base library is both a significant investment and an organisational challenge. A further example of the benefit of such a tool is the "evaluators toolkit" [42] developed for astrophysics applications. Fusion should take the lead in this radical approach, it is essential that as ITER and IFMIF are built and operated that the nuclear data required for analyses be complete and reliable. 


\section{Conclusions}

The ways in which the needs of fusion have driven the extension of nuclear data are discussed. Some differences between fission and fusion data are noted including the importance of activation. The roles of evaluation, benchmarking and validation are discussed and a radical proposal for nuclear data development proposed.

This work, supported by United Kingdom Engineering and Sciences Research Council and the European Communities under the contract of Association between EURATOM and UKAEA, was carried out within the framework of the European Fusion Development Agreement. The views and opinions expressed herein do not necessarily reflect those of the European Commission.

\section{References}

1. News Chronicle 25/1/1958.

2. N. Holtkamp, in 24th Symposium on Fusion Technology, Sept. 2006, Warsaw, Poland, Fus. Eng. Design (to be published).

3. IFMIF International Team, IFMIF Comprehensive Design Report, 2004.

4. D.J. Ward, I. Cook, Y. Lechon, R. Saez, Fus. Eng. Design 75-79, 1221 (2005).

5. I. Cook, N. Taylor, D. Ward, L. Baker, T. Hender, Culham Report UKAEA FUS 521, 2005.

6. S. Cierjacks, K. Ehrlich, in Proceedings of the International Conference on Nuclear Data for Science and Technology, Jülich, 1991 (Springer-Verlag, 1992), p. 259.

7. S. Ganesan, D.W. Muir, A.B. Pashchenko, IAEA Report INDC(NDS)-311, 1994.

8. A.B. Pashchenko, H. Wienke, IAEA Report IAEA-NDS-175 Rev. 3, 1998.

9. D.L. Aldama, A. Trkov, IAEA Report INDC(NDS)-467, 2004.

10. IRDF-2002, IAEA Technical Reports Series No. 452, Vienna, 2006.

11. R.A. Forrest, J. Kopecky, J.-Ch. Sublet, Culham Report UKAEA FUS 486, 2002.

12. J.-Ch. Sublet, A.J. Koning, R.A. Forrest, J. Kopecky, in Proceedings of the International Conference on Nuclear Data for Science and Technology, Santa Fe, 2004 (AIP 769, 2005), p. 203.

13. J. Kopecky, H. Gruppelaar, A. Hogenbirk, H.A.J. van der Kam, D. Nierop, NRG Report ECN-C-94-16, 1994.

14. A. Koning et al., OECD NEA JEFF Report 21, 2006.

15. M.B. Chadwick, P. Obložinsky et al., Nucl. Data Sheets 107, No. 12, 2931 (2006).
16. A.V. Ignatyuk, S.A. Badikov, A.I. Blokhin et al., VANT, Ser. Nuclear Constants 3-4, 59 (1997).

17. S. Chiba et al., J. Nucl. Sci. Technol. 39, 187 (2002).

18. K. Shibata et al., J. Nucl. Sci. Technol. 39, 1125 (2002).

19. R.A. Forrest, J. Kopecky, J.-Ch. Sublet, Culham report UKAEA FUS 535, 2005.

20. E.T. Cheng, D.L. Smith, in Proceedings of the International Conference on Nuclear Data for Science and Technology, Jülich, 1991 (Springer-Verlag, 1992), p. 273.

21. R.A. Forrest, Fus. Eng. Design 43, 209 (1998).

22. M.R. Gilbert, R.A. Forrest, Culham report UKAEA FUS 509, 2004.

23. M.R. Gilbert, R.A. Forrest, Fus. Eng. Design 81, 1511 (2006).

24. R.A. Forrest, Fus. Eng. Design 81, 2143 (2006).

25. A.J. Koning, S. Hilaire, M.C. Duijvestijn (these proceedings).

26. M. Herman, P. Obložinsky, R. Capote, M. Sin, A. Trkov, A. Ventura, V. Zerkin, in Proceedings of the International Conference on Nuclear Data for Science and Technology, Santa Fe, 2004 (AIP 769, 2005), p. 1184.

27. T. Belgya et al., IAEA-TECDOC-1506, Vienna, 2006.

28. P. Pereslavtsev, U. Fischer, in Proceedings of the International Conference on Nuclear Data for Science and Technology, Santa $\mathrm{Fe}, 2004$ (AIP 769, 2005), p. 215.

29. P. Pereslavtsev, U. Fischer, Nucl. Instrum. Meth. B 248, 225 (2006).

30. P. Pereslavtsev, U. Fischer (these proceedings).

31. H. Leeb, M.T. Pigni, I. Raskinyte, in Proceedings of the International Conference on Nuclear Data for Science and Technology, Santa Fe, 2004 (AIP 769, 2005), p. 161.

32. S. Tagesen, H. Vonach, A. Wallner, in Proceedings of the International Conference on Nuclear Data for Science and Technology, Santa Fe, 2004 (AIP 769, 2005), p. 79.

33. U. Fischer, P. Batistoni, Y. Ikeda, M.Z. Youssef, Fus. Eng. Design 51-52, 663 (2000).

34. P. Batistoni et al., in 24th Symposium on Fusion Technology, Sept. 2006, Warsaw, Poland, Fus. Eng. Design (to be published).

35. R.A. Forrest, J. Kopecky, M. Pillon, K. Seidel, S.P. Simakov, P. Bém, M. Honusek, E. Šimecková, Culham Report UKAEA FUS 526, 2006.

36. R.A. Forrest, J. Kopecky (these proceedings).

37. U. Fischer, P. Batistoni, E. Cheng, R.A. Forrest, T. Nishitani, in Proceedings of the International Conference on Nuclear Data for Science and Technology, Santa Fe, 2004 (AIP 769, 2005), p. 1478.

38. N. Soppera, M. Bossant, H. Henriksson, P. Nagel, Y. Rugama (these proceedings).

39. U. Fischer (these proceedings).

40. R.A. Forrest, Fus. Eng. Design 54, 387 (2001).

41. R.A. Forrest, Culham Report UKAEA FUS 484, 2002.

42. M.S. Smith et al. (these proceedings). 